\section{INJECTIONS AGAINST CHOLERA.}

\section{A Lecture}

By M. HAF F K N E.

\section{(Specially reported for THE LANCET.)}

M. HAFFKINE, who has been on a visit to this country and has made some demonstrations at Netley Hospital of his method of inoculating a modified cholera virus as a protective against cholera, was, prior to his departure to India to study r.holera in that country, invited to give at the Laboratories of the Royal College of Physicians and Surgeons a demonstrathion of his method of procedure.

On Wednesday last, Feb. 8th, a paper which he had prepared was read on his behalf by Dr. Armand Ruffer. There has been very considerable difficulty, arising from many causes, in placing the results of M. Haffkine's researches before the readers of THE LANCET. This difficulty is not in any way attributable to M. Haffkine, and has largely arisen from the excusably imperfect translation into English of his paper, which, however, has been carefully revised in the following report.

Dr. Pary having briefly introduced M. Haffkine to the large audience, Dr. Ruffer proceeded to read the paper, which was as follows :-

"The discovery that microbes may change their characters and are capable of transformation by artificial means into vaccines of fixed and ascertained power is without doubt the greatest step which has been taken in medical science since disease-producing microbes were first studied. The task which then presented itself to investigators was that of disovering the germs of contagion which were recognised as being the cause of disease and of elaborating substances protective against them.

M. Pasteur, after having discovered the first principles of this method by experiments in 'fowl cholera,' developed them greatly by his studies on anthrax; he then turned his attention to hydrophobia, which, being a disease common to both men and animals, was an appropriate one in which to employ the methods previously applied to animals in the treatment of human diseases.

"The experience acquired in the endeavour to prevent hydrophobia by inoculation-the first human disease treated according to the new method-led to the formation of a verfectly clear programme, to be followed by investigators in the direction in question. It was shown that success was to be arrived at through the preparation of a virus gradually increasing in strength accorling to a fixed scale until it reached a degree of virulence above that of the ordinary virus, which would allow of the organism becoming accustomed to the poison with which it was infected. The traits zient intensif of hydrophobia, based upon the acquisition of a virus of maximum strength, has shown the true rôle of the virus kept in a fixed state of 'exaltation' by specially appropriate experiments. The acquisition of virus of this kind and the establishment of a method suitable for keeping it in a fixed state are the ultimate aims of the research for a vaccine, since it is essential gradually to accustom the organism under treatment to this fixed virus. It is known that this task may be accomplished by the transference of infective organisms chrough a series of animals and that it is by cultivating it through a long series of such living animals that the microbe acquires the maximum of its infective power.

"In the case of cholera the first attempts towards the solution of this problem began with the infection of animals through the digestive canal. In this way the culture of the microbe took place in a part in which other microbes existed, which fact from the first rendered the means employed uncertain. The result of the introduction of a microbe into such parts depends upon the nature of the microbe. The purification of the microbe by intermediate artificial culture made in transmission between two animals is a source of diminution of virulence which counterbalances the "exaltation' obtained. This explains how MM. Pfeiffer and Nocht in seeking to strengthen the cholera microbe by passing it alternately through animals and cultures were not able to obtain a microbe capable of overcoming the natural resistance of birds; and this is the reason why M. Roux and myself (M. Haffkine), in trying to transfer the intestinal contents from one animal directly to another, according to the method used by Gamaleia, have seen our series broken after the third or fourth transference.

" The idea which M. Gamaleia had in 1888 of cultivating the microbe in the thoracic cavity of animals gave rise to considerable hope, and at one time the task was regarded as accomplished. Unhappily this method, perfectly applicable to the microbe which M. Gamaleia used - the "vibrio Metchnikovi '-was found to be defective when it was applied to the Asiatic cholera microbe, as $M$. Roux and myself ascertained in the series of trials which we have made in this direction. M. Hueppe in 1888 conceived the idea of producing infection of animals by the introduction of the cholera microbe into the peritoneal cavity ; this has since been revived by M. Pfeiffer, who stated that in the case of the cholera microbe a change was produced similar to that seen in diphtheria, tetanus and other diseases which are characterised by a local development of the living organism without an invasion of the blood or of the visceral organs. Thi change is simply that the infective agent itself disappears in the animal killed by the microbe; and if it were possible to again find the remains of the culture injected, and to transfer them to a second animal, this second animal would retain no further trace of the microbes, and the further passage would be interrupted. In the work carried out in the Pasteur Institute with regard to inoculation against Asiatic cholera the infection of the animal by the peritoneal cavity was chosen as the starting point, from which point a method has been worked out which permits of the culture of the microbe in the animal organism in a state of purity during an infinite series of generations, the exaltation of it to an ascertained maximum strength, and the possibility of keeping it at the same degree of virulence for an unlimited period of time. This method is exemplified by three series of experiments which were the subject of a publication in the Comptes - rendus le la société de Biologie of Paris. These are as follows :-

"(a) Giving the first animal a dose larger than the fatal dose and killing this animal in a sufficiently short space of time to be able easily to find the more resisting microbes.

(b) Exposing the discharge taken from the peritoneal cavity to the air for several hours.

" (c) Then transferring this discharge to the next animal, of large or small size, according to the concentration of the discharge.

The method has been verified by other experimenters with satisfactory results. The properties of the virus which is obtained in this way may be ascertained by observing that upon intra-peritoneal inoculation it always kills guinea-pig: in about eight hours, and the fatal dose for this animal is reduced to about twenty times less than that which it would have been necessary to administer for the microbe with which the experiment began. With the same inoculation it kills rabbits and pigeons by a dose which would have been harmless at the beginning of the infection experiments. It kills guineapigs when it is injected into the muscular tissue. Inoculation under the skin causes the formation of a large swelling, which produces sloughing of that part of the skin and the formation of a gaping wound which is curable in two or three weeks. This is the basis of my researches.

"The virus when injected under the skin of a healthy animal gives the creature, after several days, immunity from the poison of cholera, however it may be conveyed to it. If an attempt be made to infect an animal which has been so treated, either by Koch's method through the alimentary canal, by neutralisation of the gastric juice and the injection of opium into the peritoneum, by the introduction of the microbe into the intestines by the method of Nicati and Rietsch, through the muscles, or by (the most fatal of all) intra-peritoneal injection, the animal resists, whilst the control animals are killed. Inoculation of animals against cholera in this manner has been definitely established. But the operation thus described cannot be applied to man. The wound following on the injection under the skin is alarming to look at, and is in all probability painful. Besides, although it does not in itself present any danger to the health of the individual, it subjects him to all the dangers arising from an open wound. By growing it at a temperature of $39^{\circ} \mathrm{C}$. and in an atmosphere freely exposed to the air this corrosive action is removed from the microbe. Under these conditions the first crop of the cholera microbes die rapidly, say in from two to three days; but others are sown again in new centres immediately before the death of the first. After a series of generations of 
this kind a culture is obtained which, if injected under the skin of animals even in very large doses, produces merely a temporary oedema and so prepares the inoculated organism that the injection of this modified virus of fixed exalted vaccine produces only a local reaction of the slightest kind. Thus the method of inoculation deals with two raccines-a mild one obtained by weakening the 'fixed virus,' and a 'strengthened vaccine' which is really the exalted virus itself. The reason an ordinary virus is not used to obtain the weakened vaccine, but one the nature of which has been previously ascertained in the laboratory, is that the viruses in their natural state, especially when they have reached a saprophytic stage of development, present such great differences that there is no certainty in their application. This recalls to mind the story of inoculation for small-pox. The mildness or the severity of an infection does not depend merely on the real strength of the contagious substance, but upon the resistance of the individual from whom the poison has been taken. Thus in taking vaccine for use as vaccine lymph from a subject slightly affected a very weak substance was sometimes obtained incapable of causing protection, but still able to kill individuals less resistant. The great point of Jenner's discovery lay in the fact that it indicated a substance fixed by successive passages through animals at a virulence above that which is fatal to human beings. Another example is given in the old method of inoculation against antbrax by Toussaint, the first of its kind, which has had to give way to the method of M. Pasteur simply because Pasteur's method was based upon a virus of fixed strength and produced results with certainty, conditions which were wanting in the former method. In $1885 \mathrm{Dr}$. Ferran of Barcelona, with the object of preserving the population of the Peninsula from cholera, injected his patients with the ordinary virus taken from dead bodies and cultivated in the laboratory. The statistics of the results obtained by him were so uncertain that it could not be recommended. The fundamental feature of the Pasteurian method was the possibility of treating the animal organism by vaccines of an absolutely fixed nature by means of special operations. That was the whole secret and the sole guarantee of the success of its application.

"The method of inoculation against cholera worked out by experiments on guinea-pigs was afterwards tried upon rabbits and pigeons before it was applied to man. These animals were chosen in order to secure very differently organised subjects, and to obtain the power of generalising the conclusions before extending the experiments to human beings. The same results were obtained on all these animals and it was decided to apply the operation to man. The symptoms produced by this operation have been described in several scientific publications. The method has been tried at Paris, at Cherbourg and at Moscow on about fifty persons of both sexes, between the ages of nineteen and sixty-three, of French, Swiss, Russian, English and American nationalities. [M. Haffkine was the first to be inoculated with his own virus. He has undergone the operation three times.]

"In all cases the method has proved to be absolutely harmless to health. The symptoms consisted merely of a rise of temperature, a local sensitiveness at the place of inoculation and the formation of a temporary swelling at the same place. The symptoms appear about two or three hours after inoculation-viz., fever and general indisposition, which disappear in from twenty-four to thirty-six hours, and sensitiveness and cedema lasting three or four days. The symptoms following the second inoculation are usually more marked but of shorter duration; the whole giving rise to a feeling as of a bad cold in the head, lasting from one to two days. The microbes which are introduced under the skin do not propagate, but die and disappear after a certain time. It is the substances which they contain which being set free on the death of the animals act upon the animal organism and confer immunity upon it. It is found that the same result can be obtained if the microbes be killed before inoculation. Thus raccines have been preserved in weak solutions of carbolic acid in which the microbes die at the end of several hours, and the vaccine preserved in this way has been found to be still efficacious six months after its preparation. There is evidently considerable advantage in preserving the microbes in this way. It enables them to be used by persons having no bacteriological training, and the absence of living germs makes the vaccines perfectly safe. The carbolic acid that they contain preserves them against any invasion of other microbes, and as they can be kept for several months their preparation may be carried out at a central laboratory whence the vaccine ampoules can be sent out to operators. But it is probable that the immunity given by these preservec vaccines does not equal in persistency that produced by living germs, and as the method is not yet supported by verified statistics it is better that inoculations should be made as much as possible with living virus, so as to secure the most reliable results.

"As to the length of time that immunity so produced wil? last, there were no animals at the laboratory of the Pasteur Institute that had been inoculated for a period longer thar four months and a half. At the end of that time their immunity was found to be perfect and was likely to last much longer. The experiments upon man, added to the hundreds of experiments which had been made upon animals, testify to the absolutely harmless nature of these operations, and there is no difficulty in proving their efficacy by experiment independently of the species of animal which may be employed.

"On Wednesday last Dr. Woodhead inoculated six animals with the first vaccine. The second inoculation was made two days ago. Yesterday, in presence of Dr. Woodhead and several other gentlemen, these six animals were inoculated with 6 c.c. of virus of the cholera culture into the peritoneum. At the same time six other animals, which had not been inoculated before, were injected with half the dose which the first set of animals had received. The result is that the inoculated animals are to-day perfectly well. To any one accustomed to the clinical appearance of guineapigs after inoculation the animals you see before you have a perfectly normal appeasance. On the other hand, of the six animals which were inoculated with half of the dose, two are already dead, two are very ill, and there is not the slightes doubt that the two others will be dead to-morrow.

"It is unnecessary to say that we cannot perform a simila" experiment on man, although this would be the only means by which a definite experimental demonstration could be given. This being the case, evidently the objection might be raised that, although this vaccination renders guinea-pigs, rabbits and pigeons very resistant, yet is might be possible that man might escape this law. That, indeed, is not impossible, and-we lay stress, much stress, upon the fact-it is by experiment that our method must be established. It is by direct application and experi. ment that we must verify to ourselves and others the true value of the means extolled. Yet, after the experience already acquired, we think there is little probability that negative result will be obtained. The difference between a mammal like the rabbit or the guinea-pig and a bird like the pigeon, from an anatomical and physiological point of view, is much greater than between rodents and man. "Hence, when the same law is proved to exist amongst animals so different there is little probability that man is not also subject to it. The symptoms following on inoculation of our vaccines are analogous in all the animals experimented on, in cluding man. The same changes of temperature take place in all. In the animals which have been rendered resistant against cholera immunity is evidenced by the fact that every subcutaneous inoculation, made even with weakened virus, induces a rise of temperature greater than the preceding. The same thing takes place in man. We hope that the parallel will extend to this modification of the organism which pro duces the resistance against an infective agent. But the sole and definitive confirmation of this hope can be obtained only by the application of the method where cholera makes ravages amongst the human race."

Guinea-pigs were exhibited to show the effects on the animals when inoculated under the skin and when inoculated into the peritoneal cavity. The difference between the effects of the strong virus and the weak virus was also demonstrated. One of the animals was inoculated on Saturday last with one-tenth part of the tube of the weak vaccine. If they would examine it, they would find that it had, at the point of inoculation, a smal! localised cedema, but there was nothing resembling abscess or anything of the sort. It would disappeas in a very few days. On the other hand, another animal wa. inoculated on Saturday at the same time, subcutaneously also, with the stronger vaccine, and they found that a very definite little abscess was formed, that the skin was necrosed over it: surface, and that it would take some time to heal. Anothe? animal inoculated with the same quantity of virus int the peritoneal cavity died within from sixteen to twenty four hours. The experiment on the one which had re 
ceived the weak vaccine would be continued. The result would be that when it was inoculated again the abscess as shown in the other animal would not form. That was the reason why these two vaccines were used in inoculating man. The first produced a slight oedema, but it did not produce an abscess, and when they used the strong virus after the weak one, instead of producing an abscess, they simply got a small swelling which disappeared in a few days. Since M. Haffkine had been in England two gentlemen, one of whom had proceeded into a dangerous part of Persia, the other being a medical officer of health near London, had been inoculated by him. The following is a clinical report on one of these gentlemen, taken by his son, who is a medical man and a pathologist. The notes were as follows:- "The first inoculation was made on Feb. 4th, 1893. The gentleman in question is fifty-six years old. Temperature at the time of inoculation $97^{\circ} \mathrm{F}$. under the axilla. After three hours slight lassitude set in. At 5 P.M. slight pain in the side; temperature $98^{\circ} \mathrm{F}$. in the mouth; seat of inoculation red, indurated, two inches by two. At 7 P.M., temperature $99^{\circ} \mathrm{F}$. At 9 P.M. lassitude increased; took a slight dinner. The son notices that his father looks markedly febrile, feels tired and goes to bed; temperature $99^{\circ}$; pulse increased to about 80 ; feels slightly cold and there is slight pain in the limbs. At 10.30 slept well since going to bed; temperature $99^{\circ}$; respiration above normal. Soon went to sleep again and passed very good night. Feb. 6th, 9 A.M., temperature $98^{\circ}$; appetite not so good as usual ; was quite able to resume usual duties; symptoms by no means severe; the most remarkable phenomena are the onset and the feeling of lassitude and sleepiness. At 11.40 , temperature $99^{\circ}$. Feb. 6 th, morning temperature $98^{\circ}$, evening $97^{\circ}$. Feb. 8th, morning temperature $97^{\circ}$; evening $97.5^{\circ}$. Feb. 8th, morning temperature $975^{\circ}$. The second inoculation was made to-day." The notes in the case of the other gentleman had unfortunately been lost, but practically the same result ensued. This gentleman summed up his experience by declaring that he would Tather be inoculated against cholera than be vaccinated again. M. Haffkine made a post-mortem examination of one of these inoculated animals that had succumbed to the intraperitoneal injection of the exalted virus, and the pathological appearances as shown in guinea-pigs were demonstrated. There was a red injected spot showing that a portion of the inoculation had entered the muscular tissue. The abdomen was distinctly distended and contained serous fluid. The bowels were red and injected much more than is usual in guinea-pigs. All the other organs were normal. On the liver, between the diaphragm and that organ, slight traces of lymph could be seen. The spleen, if the animal lives
for a certain length of time, is slightly enlarged; in this case the spleen was distinctly enlarged, the animal having lived twenty-four hours. The cultures from which all these experiments were made were from a case of cholera which occurred in Cochin China, and were forwarded direct to M. Haffkine.

M. Haffkine then made a cover-glass preparation of the cedematous fluid from the peritoneal cavity to show that it contained a pure culture of the cholera bacillus, and this was examined with much interest by many who were present.

Dr. Pary at the termination of the proceedings expressed the thanks of the audience to $M$. Haffikine for the demonstration, characterising it as an act of courtesy and kindness in him to remain in England solely for the purpose of giving this demonstration. It was sincerely to be hoped that the promise afforded by his observations which were now being conducted would be realised, and that we might Whave an illustration of the power of knowledge in the bringing ander subjection of one of those diseases which formed a great terror to mankind. Turning to M. Haffkine, he said: "We desire to express our gratitude to you for having remained here in England to afford us an opportunity of witnessing this demonstration, and I would ask Dr. Ruffer, who has served us so well as a medium between yourself and us, to play the opposite part and serve as a medium between us and $M$. Haffkine by conveying to himo the thanks of the meeting." This Dr. Ruffer did in felicitous terms, and the demonstration then concluded.

Literary INTelltgence. - Mr. H. K. Lewis announces the issue at an early date of a second edition of Dr. Radcliffe Crocker's work on Diseases of the Skin, which has been greatly enlarged and brought up to date. A new volume of Lewis's Practical Series is also nearly ready, and is entitled "Public Health Laboratory Work," by H. R Kenwood, M.B., D.P.H.

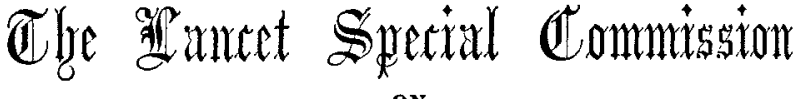

\author{
ON
} SEAMEN'S DIETARIES.

CoNSIDERABLE attention has been drawn during the past three or four months to the food of British merchant seamen and a committee consisting of the representatives of many of the shipowners' associations has been investigating the question with the object of ascertaining in what respects a revision of the scales now in vogue is advisable. We have independently made many inquiries from representative seamen themselves, and have thought it well to lay their views before the profession when comparing the present dietaries and that proposed for future adoption in the merchant service with some dietaries laid down for representative bodies of landsmen, so that the question may be studied from as many sides as possible. We may say at once that very few complaints have been made to us as to the quality of the articles supplied to sailors, with the exception of tea and coffee, which were often stated to be of very inferior quality. There is an almost unanimous opinion that the quality of the staple articles of beef, pork bread and flour has immensely improved in late years. The quantities fixed by the minimum scale are said to be not too great for protracted and arduous work, and to be far too small in the special long voyage sailing ship scale. It is generally supposed that the Board of Trade fixes the amount and examines into the quality of the food supplied to the sailor but this is not the case, for in the form of agreement issued by the Board of Trade no detailed scheme of dieting is given: there is only found a skeleton scale of the provisions to be allowed and served out to the men during the voyage for which the agreement is made.

This skeleton scale (Table I.) must be filled in before the agreement is signed, and the quantity and quality of provisions supplied are therefore solely a matter of contract between the owner and the crew. But practically a regular scale is inserted into the articles, and when the sailo signs these with the "usual" diet scale filled in, he clearly understands by that term that the minimum scale of 1854 (Table II.) is intended, and this is deemed a sufficient one by the authorities. It is stated by the shipowners that this scale is rarely adhered to, and this is quite true in many cases, but from our inquiries amongst the sailors it is clear that it is sometimes most rigidly followed; and it is obvious that if it is compulsorily adhered to nothing in addition can be insisted on, and that no objection can be taken by the sailor after he has signed articles. We must therefore take it into consideration as being the actual basis on which alone the seaman can insist, and which in many ships is all that he will get during a voyage.

The absence of any preserved meats or,'vegetables and the monotony of a diet practically limited to bread, salt beef and pork, with soup once and dough three times a week, at once shows that such a scale is only fitted for short voyages or for voyages in which the ship is not too long from port to port where the substitutes of fresh meat and vegetables can be often made use of. The supplying of these substitutes should not be optional at the master's discretion they should be compulsory whenever the ship arrived at a port. The shipowners clearly recognise - that this diet is deficient in fresh provisions, for a special longvoyage scale (Table III.) is also in use, and in this preserved meats, potatoes and vegetables, raisins, preserves and molasses are included. This scale is agreed to by the sailor also when he signs his agreement, and he is quite conversant with its particulars before he starts on a long voyage.

A comparison of the two dietaries shows that in the long voyage scale the seaman loses per week $1 \frac{3}{4} \mathrm{lb}$. of bread, $2 \frac{1}{2} \mathrm{lb}$. of beef and $1 \frac{3}{4} \mathrm{lb}$. of pork, and gains $2 \mathrm{lb}$. of preserved meat, a quarter of a pint of soup and half a pound of flour. The preserved potatoes (6oz.), preserved vegetables ( $1 \mathrm{lb}$.), coffee $\left(1 \frac{3}{4}\right.$ oz. $)$, raisins ( 3 oz. $)$, suet ( 2 oz. ), butter $\left(\frac{1}{2}\right.$ oz. $)$, preserves (11b.) and molasses (half a pint), although excellent varieties in a dietary and most useful as aids in the prevention of scurvy, can hardly be considered as efficient substitutes for the above-mentioned loss of the more nutritious articles-biscuits, beef and pork. In fact, instead of being called substitutes, all these antiscorbutics should 